\title{
EVOLUÇÃO DA TEXTURA CRISTALOGRÁFICA DA AUSTENITA COM A FORMAÇÃO DE MARTENSITA DURANTE A LAMINAÇÃO A FRIO DE AÇO INOXIDÁVEL AUSTENÍTICO 20 ILN
}

\author{
Camila Oliveira de Souza' \\ Carolline Serafim da Silva ${ }^{2}$ \\ Juciane Maria Alves ' \\ Andersan dos Santos Paula' \\ Luiz Paulo Mendonça Brandão' \\ Saulo Brinco Diniz ${ }^{1,3}$ \\ Isabela Santana de Oliveira ${ }^{4}$
}

\section{Resumo}

O material em estudo consistiu em um aço inoxidável austenítico 20ILN, na forma de chapa, na condição laminada a quente. A textura cristalográfica da austenita foi medida antes e após a laminação a frio em duas condições: a temperatura ambiente e pré-resfriada em salmoura. Com o aumento do percentual de deformação há uma redução na fração de austenita para formação da martensita, resultando na intensificação das componentes de textura pré-existentes (fibras $<1 \mid 4>/ / \mathrm{DL}$ e $<221>/ / \mathrm{DL}$ em $\phi=20$ e $70^{\circ}$, com destaque para as respectivas componentes $\{1 \mid 4\}<100>$ e $\{22 \mid\}<|| 0\rangle$ ) e desenvolvimento de novas componentes $\{|| 4\}<|43>,\{\mid 14\}<| 1|0\rangle,\{22 \mid\}<0 \mid 3>;\{22 \mid\}<023>$ e $\{22 \mid\}<\mid 22>$, pertencentes às fibras $<|| \mid 4>/ / D L$ e $<22 \mid>/ / D L$ da fase austenítica.

Palavras-chave: Aço Inoxidável Austenítico; Laminação a frio; Austenita; Textura cristalográfica.

\section{AUSTENITE CRYSTALLOGRAPHIC TEXTURE EVOLUTION IN MARTENSITE FORMATION DURING COLD ROLLING OF 2OILN AUSTENITIC STAINLESS STEEL}

\begin{abstract}
The material studied consisted of a 20ILN austenitic stainless steel, in sheet form, in the hot rolled condition. The austenite crystallographic texture before and after a cold rolling in two conditions: at room temperature and pre-cooled in brine. With the increase of the deformation there is a reduction in the austenite fraction and increasing of the martensite fraction, resulting in the intensification of the pre-existing texture (fibers $<1 \mid 4>/ / R D$ e $<221>/ / R D$ in $\phi=20$ e $70^{\circ}$, with emphasis on the respective components $\{1 \mid 4\}<100>$ and $\{221\}<110>$ ) and development of new components $\{1 \mid 4\}<|43>,\{1 \mid 4\}<| 10>,\{22 \mid\}<0 \mid 3>$; $\{22 \mid\}<023>$ and $\{221\}<\mid 22>$, belonging to the fibers $<|| 4>/ / R D$ and $<22$ I $>$ //RD of the austenitic phase.
\end{abstract}

Keywords: Austenitic Stainless Steel; Cold Rolling; Austenite; Crystallographic texture.

\section{INTRODUÇÃO}

Os aços inoxidáveis austeníticos (AIAs) possuem grande relevância, pois aliam excelente resistência à corrosão, soldabilidade e elevadas propriedades mecânicas [I]. De acordo com a composição química dos
AlAs, estes podem apresentar uma característica particular: Transformação de fase induzida por plasticidade, efeito TRIP (ou do Inglês, Transformation Induced Plasticity), em que a austenita se transforma em martensita através

'Instituto Militar de Engenharia - IME, Rio de Janeiro, RJ, Brasil. E-mail: camilaosouza@oi.com.br

${ }^{2}$ Galvasud, Porto Real, RJ, Brasil.

${ }^{3}$ Centro Federal de Educação Tecnológica - CEFET, Angra dos Reis, RJ, Brasil.

${ }^{4}$ Universidade Federal Fluminense - UFF, Volta Redonda, RJ, Brasil. 
da aplicação de uma tensão ou deformação [2]. Com o objetivo de se obter um aço com um custo inferior e que pudesse ser utilizado em certas aplicações, desenvolveu-se a série 200 de sistema $\mathrm{Fe}-\mathrm{Cr}-\mathrm{Mn}-\mathrm{N}$, com adições reduzidas de níquel e adição combinada de nitrogênio e manganês como seus substitutos na função de preservar a estrutura austenítica [3].

Para a maioria dos AlAs, os quais possuem baixos ou moderados valores de Energia de defeito de empilhamento (EDE), a textura cristalográfica esperada é do tipo Brass que é desenvolvida pelos mecanismos de deformação, como maclação mecânica e formação de bandas de cisalhamento [4]. Quando se refere à evolução da textura cristalográfica do AIAs 20I, verifica-se um estudo recente de Souza et al. [5] que relataram a evolução nas componentes de textura da austenita deformada, encontradas neste material, quando submetido à laminação a frio. As componentes que foram observadas para austenita deformada foram: Goss $\{0 \mathrm{II}\}$ $<100>$, Brass $\{01 \mathrm{I}\}<2 \mathrm{II}>$, pertencentes à fibra $\alpha \mathrm{CFC}$, e $S\{123\}<634>$, para menores níveis de deformação $(\varepsilon=0,22$ associada a um pouco mais de $70 \%$ de austenita não transformada, e $\varepsilon=0,5 \mathrm{I}$ associada a um pouco mais de $50 \%$ de austenita não transformada). Notou-se que acima de 0,5 I de deformação verdadeira, há manutenção da fração de austenita não transformada, todas as componentes previamente observadas se fortaleceram, a fibra $\alpha$ CFC se tornou mais nítida e a componente Brass atingiu uma intensidade máxima.

Por outro lado, um estudo também recente relatado por Souza et al. [6], fez uso de um aço 20ILN submetido à laminação a frio sob duas condições: $A$ temperatura ambiente e com pré-resfriamento em salmoura antes da laminação, com o objetivo de avaliar a formação de martensita e evolução da resistência mecânica do material. As técnicas de caracterização utilizadas foram macro Dureza Rockwell C (HRC), Ferritoscopia e Difração de Raios-X (DRX). Os resultados mostraram que tanto a fração volumétrica de martensita formada, como a dureza, foram maiores nas amostras submetidas a maiores percentuais de redução e pré-resfriadas em salmoura. Diferentemente de Souza et al., no trabalho de Souza et al. a saturação na formação de martensita se dá em percentuais mais baixos de deformação verdadeira e de martensita formada. Este comportamento é provavelmente associado aos maiores percentuais de $\mathrm{Ce}$
$\mathrm{N}$ que favorecem muito mais o encruamento da austenita pelo incremento na EDE e decréscimo da temperatura de início da transformação martensítica $\left(\mathrm{M}_{\mathrm{i}}\right)$ e da temperatura que prevê a formação de $50 \%$ de martensita devido a $30 \%$ de deformação verdadeira $\left(M_{\mathrm{d} 30}\right)$.

No presente trabalho, fazendo uso do mesmo material de Souza et al. [6], o objetivo consistiu em avaliar a evolução cristalográfica da fase austenítica das amostras nas condições como recebida (CR), laminada a quente, e laminada a frio (a temperatura ambiente e pré-resfriadas em salmoura) em função da formação de martensita induzida por deformação em temperaturas distintas.

\section{MATERIAIS E MÉTODOS}

O material em estudo consistiu em um aço inoxidável austenítico $20 \mathrm{ILN}$, na forma de chapa com aproximadamente $5 \mathrm{~mm}$ de espessura na condição laminada a quente, fornecido pela empresa APERAM South América e composição definida por: $\mathrm{Cr}=17,0043 \%, \mathrm{Mn}=6,9078 \%, \mathrm{Ni}=4,0093 \%$, $\mathrm{Si}=0,3088 \%, \mathrm{Cu}=0,0239 \%, \mathrm{Mo}=0,0188 \%, \mathrm{~N}=0,2000 \%$, $\mathrm{C}=0,03 \%$, percentual em massa.

Este aço foi laminado a frio em duas condições distintas (temperatura ambiente e pré-resfriado a cada passe em salmoura gelada, de aproximadamente $-5^{\circ} \mathrm{C}$ ). Estes corpos de prova foram laminados com 4,6 e 8 passes ( $10 \%$ de redução na espessura a cada passe). As respectivas deformações verdadeiras e fração de martensita medidas por ferritoscopia, em trabalho prévio [6], são mostrados na Tabela I.

A medição da textura cristalográfica foi realizada através de um difratômetro de raios-X PANalytical X'Pert Pro MRD com os seguintes parâmetros: Passo angular phi e psi de $5^{\circ}$, anodo de cobalto, filtro de ferro e detector PIXcel. Para a fase austenita, que é CFC, foram analisados os planos (I I I), (200) e (220), que difratam nos respectivos ângulos $2 \theta$ de $50,91^{\circ}, 59,48^{\circ}$ e $89,25^{\circ}$, de acordo com a Lei de Bragg. Os resultados experimentais foram corrigidos utilizando o software popLA até a obtenção das curvas de função de distribuição de orientações (ODF's) em notação de Bunge.

Tabela I. Deformações verdadeiras ( $\varepsilon$ ) para os corpos de prova laminados no presente estudo

\begin{tabular}{|c|c|c|c|}
\hline Número de Passes & $\begin{array}{c}\text { Nome da Amostra - Temperatura } \\
\text { prévia a cada passe }\end{array}$ & Fração de Martensita (\%) [6] & Deformação verdadeira $(\varepsilon)$ \\
\hline \multirow[t]{2}{*}{4} & 4TA - Temperatura ambiente & 11,24 & $\varepsilon=0,38$ \\
\hline & 4SA - Salmoura gelada & 17,70 & $\varepsilon=0,42$ \\
\hline \multirow[t]{2}{*}{6} & 6TA - Temperatura ambiente & 20,64 & $\varepsilon=0,60$ \\
\hline & 6SA - Pré-resfriada em salmoura gelada & 26,41 & $\varepsilon=0,61$ \\
\hline \multirow[t]{2}{*}{8} & 8TA - Temperatura ambiente & 26,26 & $\varepsilon=0,8 \mathrm{I}$ \\
\hline & 8SA - Pré-resfriada em salmoura gelada & 30,71 & $\varepsilon=0,8 \mathrm{I}$ \\
\hline
\end{tabular}




\section{RESULTADOS E DISCUSSÃO}

Na Figura I é mostrada a ODF com as seções Phi2 em 0 e $45^{\circ}$ (notação de Bunge) da amostra na condição como recebida, ou seja, laminada a quente e 100\% austenítica. Observou-se que o material laminado a quente (Figura I) apresenta uma fraca textura preferencial, condizente com aços inoxidáveis austeníticos, por não possuírem transformação de fase durante $\circ$ tratamento termomecânico. É possível notar a formação de fibras < | | $4>/ / D L$ e $<22$ I $>/ / D L$ em $\Phi_{1}=20$ e $70^{\circ}$, com destaque para as respectivas componentes $\{\mid 14\}<\mid 00>$ e $\{22 \mid\}<|| 0\rangle$. Estas componentes são distintas daquelas observadas por Souza et al. [5].

A evolução textural da austenita deformada nas amostras laminadas a frio sob condições distintas é apresentada nas Figuras de 2 a 7. Observou-se que na seção Phi2 $45^{\circ}$ das amostras deformadas há um leve reforço nas fibras próximas a $\phi_{1}=20$ e $75^{\circ}$.

Para todas as amostras laminadas há uma forte intensificação das fibras $<|| 4 \mid / / D L$ e $<22 \mid>/ / D L$, herdadas da chapa na condição como recebida. $O$ incremento na deformação verdadeira resultante ao longo do processamento fortaleceu a dispersão das componentes cristalográficas destas fibras ou, em outras palavras, promoveu o fortalecimento destas fibras cristalográficas. Esse efeito pode ser mais evidente nas amostras laminadas a temperatura ambiente. Por outro lado, o aumento deste reforço incrementa levemente com - aumento da deformação verdadeira para a laminação a temperatura ambiente, e isto ocorre de modo oposto na condição pré-resfriadas em salmoura com semelhante de componentes com a condição a temperatura ambiente. O plano cristalográfico (III) está $35^{\circ}$ distante do plano (I I4) e $16^{\circ}$ distante do plano (22I).

Dessa forma, a fibra $<22$ I $>/ / D L$ possibilita melhor comportamento em deformação do que a </|4 //DL. Como o aumento do grau de deformação, houve um sensível fortalecimento da fibra $<22$ I $>/ / D L$ e implicou na redução da fração de austenita, que resulta no decréscimo na intensidade dos polos máximos, mas sem aparentes mudanças nas componentes de textura, conforme esperado. No entanto, as componentes desenvolvidas são distintas daquelas observadas no trabalho de Souza et al. [5], que se justificam pela herança das componentes já presentes no material como recebido (laminado a quente).

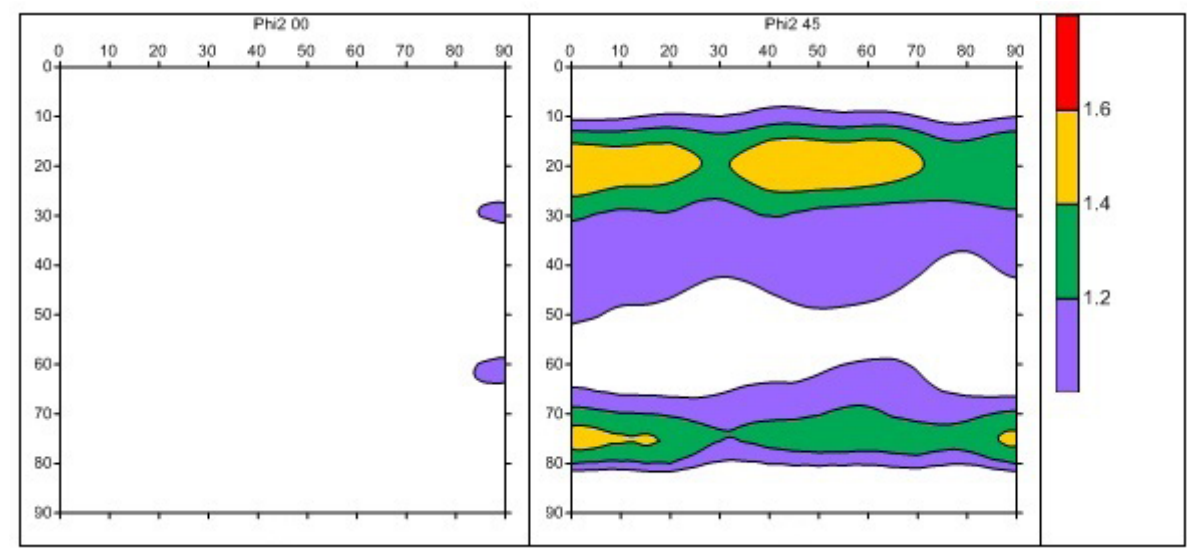

Figura I. ODF da austenita, amostra na condição como recebida com as seções Phi2 $0^{\circ}$ e $45^{\circ}$.

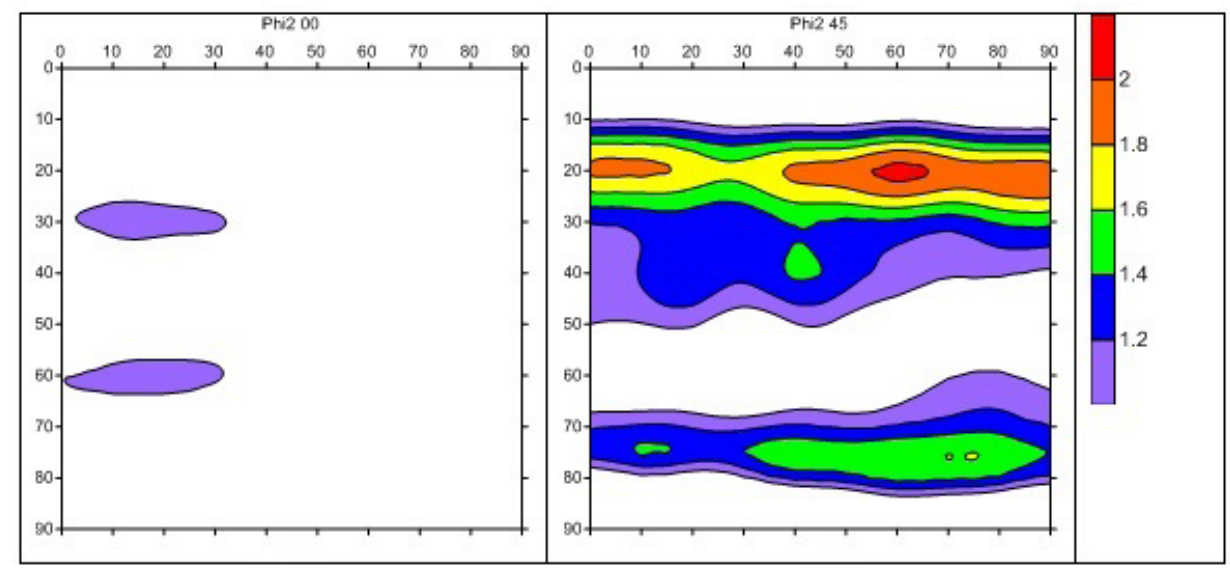

Figura 2. ODF da austenita deformada da amostra 4TA, Phi2 $0^{\circ}$ e $45^{\circ}$. 


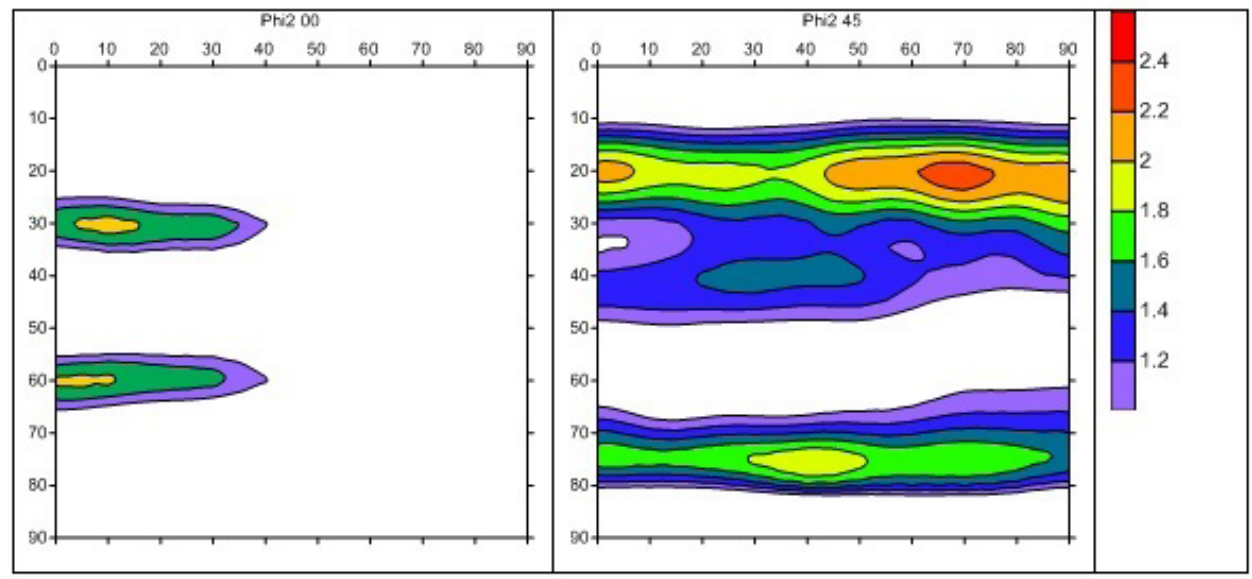

Figura 3. ODF da austenita deformada da amostra 4SA, Phi2 $0^{\circ}$ e $45^{\circ}$.

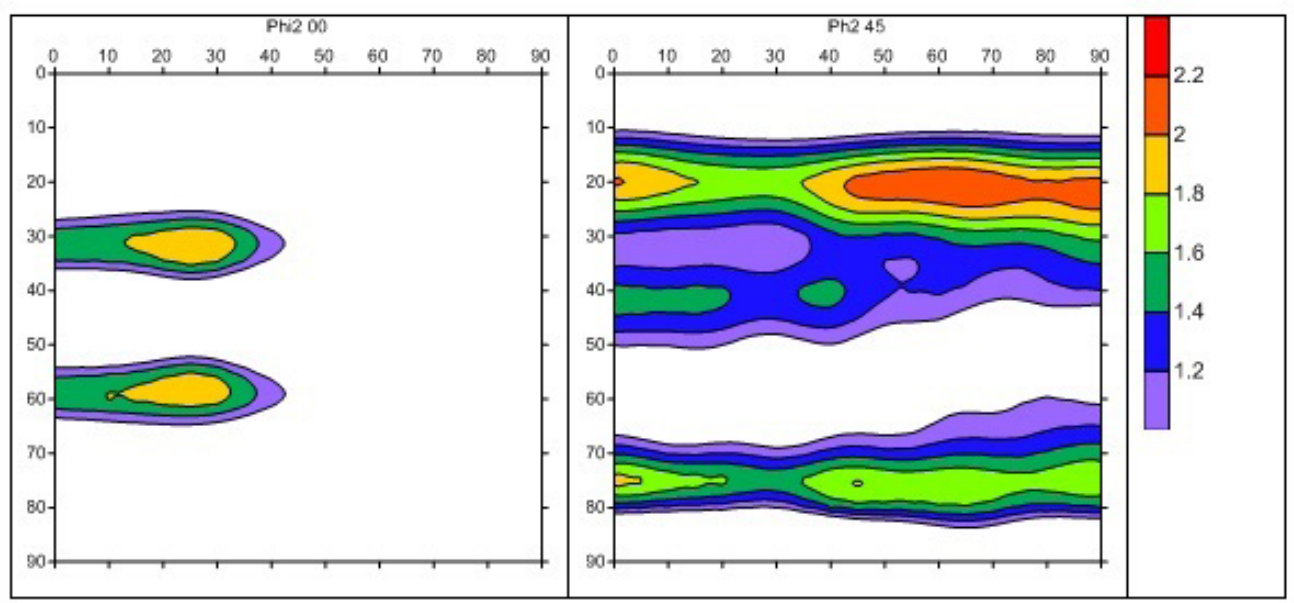

Figura 4. ODF da austenita deformada da amostra 6TA, Phi2 $0^{\circ}$ e $45^{\circ}$.

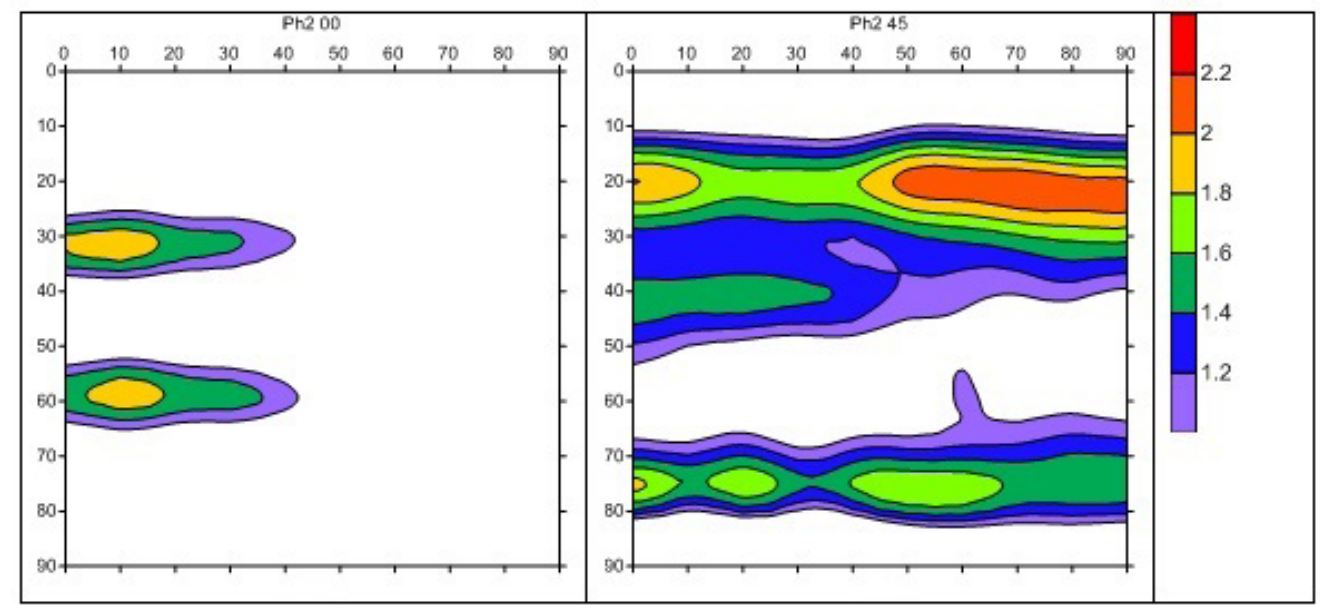

Figura 5. ODF da austenita deformada da amostra 6SA, Phi2 $0^{\circ}$ e $45^{\circ}$. 


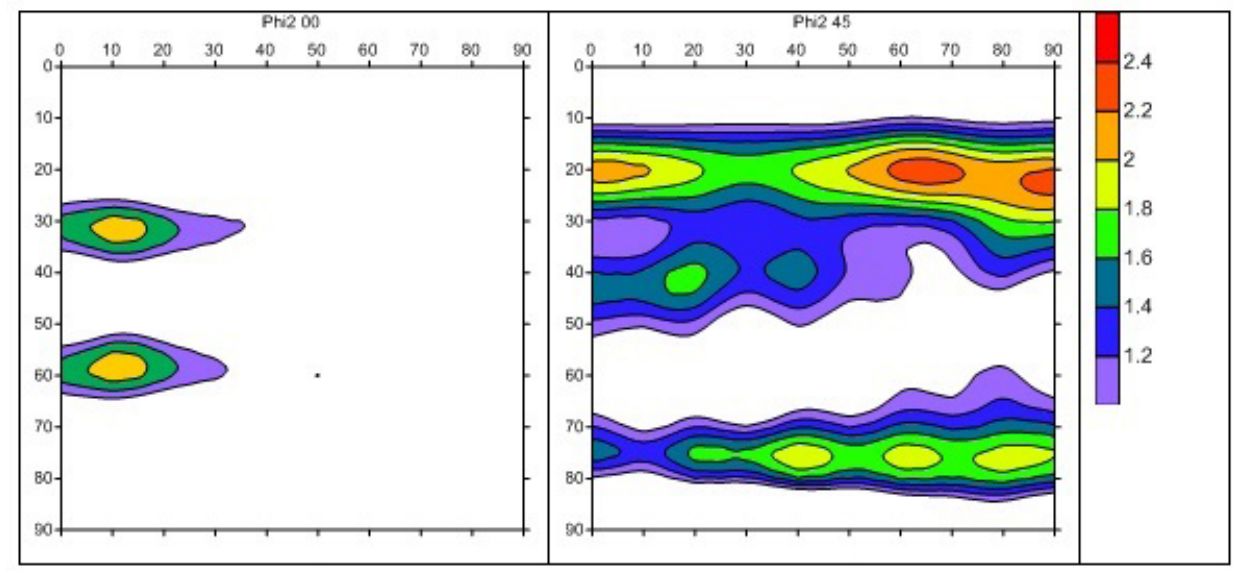

Figura 6. ODF da austenita deformada da amostra 8TA, Phi2 $0^{\circ}$ e $45^{\circ}$.

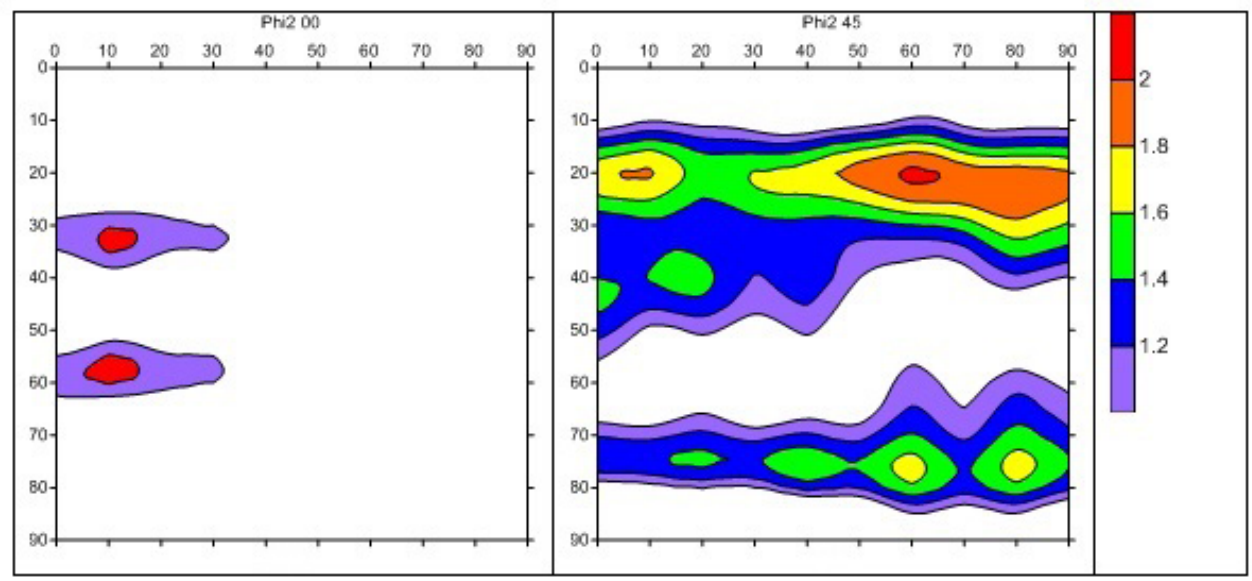

Figura 7. ODF da austenita deformada da amostra $8 \mathrm{SA}, \mathrm{Phi} 20^{\circ}$ e $45^{\circ}$.

Os resultados de evolução textural indicam que há uma intensificação das componentes $\{1 \mid 14\}<|43\rangle$, $\{|| 4\}<|| 0\rangle,\{22 \mid\}<0 \mid 3>$; $\{22 \mid\}<023>$ e $\{22 \mid\}<\mid 22>$, pertencentes às fibras citadas $<1|4| / / \mathrm{DL}$ e $<22 \mid>/ / \mathrm{DL}$, pois o material passa a ter uma orientação preferencial.

\section{CONCLUSÃO}

Conclui-se que a condição inicial, laminada a quente, tem fraca textura preferencial, sendo possível notar a formação de fibras $<|| \mid 4>/ / D L$ e $<221>/ / D L$ em $\phi_{1}=20$ e $70^{\circ}$, destacando-se as respectivas componentes $\{1 \mid 1\}\}<100>$ e $\{22 \mid\}<|| 0\rangle$. Com o aumento da deformação, há uma redução na fração da austenita e consequente decréscimo na intensidade dos polos máximos, mas sem significativas mudanças nas componentes da textura associadas às fibras < ||4 $>/ / D L$ e $<22 \mid>/ / D L$. No entanto, para as amostras laminadas a temperatura ambiente como para as amostras pré-resfriadas, com um mesmo percentual de deformação, as componentes de textura da austenita parecem ter semelhança.

\section{Agradecimentos}

Os autores agradecem ao Conselho Nacional de Desenvolvimento Científico e Tecnológico (CNPq) pela bolsa de doutorado, processo de número $|4| 57|/ 20| 5-2$ (Ano 20I5), e de produtividade a pesquisa (PQ-2), processo 307798/20 I 5-I. Como também agradecem à Aperam South America pela doação do material em estudo.

\section{REFERÊNCIAS}

I Okayasu M, Fukui H, Ohfuji H, Shiraishi T. Strain-induced martensite formation in austenitic stainless steel. Journal of Materials Science. 20I3;48:6157-6166.

2 lawamoto T, Tsuta T, Tomita Y. Investigation on deformation mode dependence of strain-induced martensitic transformation in trip steels and modelling of transformation kinetics. Vol. 40. USA: Elsevier Science; 1998. p. I73-I82. 
3 Hamada AS, Karjalainen LP, Misra RDK, Talonen J. Contribution of deformation mechanisms to strength and ductility in two $\mathrm{Cr}-\mathrm{Mn}$ grade austenitic stainless steels. Materials Science and Engineering A. 20I3;559:336-344.

4 Kumar BR, Singh AK, Mahato B, De PK, Bandyopadhyay NR, Bhattacharya DK. Deformation-induced transformation textures in metastable austenitic stainless steel. Materials Science and Engineering A. 2006;429:205211 .

5 Souza IR Fo, Zilnyk KD, Sandima MJR, Bolmaro RE, Sandima HRZ. Strain partitioning and texture evolution during cold rolling of AISI 20 I austenitic stainless steel. Materials Science and Engineering A. 2017;702:16I-I 72.

6 Souza CO, Silva CS, Alves JM, Paula AS, Brandão LPM, Diniz SB, et al. Efeito do pré-resfriamento em salmoura na formação de martensita durante a laminação piloto de aço inoxidável austenítico 20 ILN. In: Associação Brasileira de Metalurgia, Materiais e Mineração. Anais do $72^{\circ}$ Congresso Anual da ABM; 2017 Outubro 2-6; São Paulo, Brasil. São Paulo: ABM; 2017. p. 1525-1535.

Recebido em: 20 Nov. 2017

Aceito em: 07 Out. 2018 